\title{
A Fuzzy Decision Support System for Drawing Directions from Purchasing Portfolio Models
}

\author{
Davide Aloini, Riccardo Dulmin, and Valeria Mininno \\ University of Pisa, Pisa, Italy \\ \{davide.aloini, riccardo.dulmin, valeria.mininno\} @dsea.unipi.it
}

\begin{abstract}
This work presents a decision support system (DSS) enhancing users to effectively integrate classical purchasing portfolio approaches with additional strategic oriented priorities and information in order to effectively support the definition of purchasing directions and action plans.

With these aims, a fuzzy-based DSS is designed and implemented. The decision process gets inputs from the Kraljic $(\mathrm{K})$ matrix and draws directives on the traditional Olsen-Ellram (O-E) portfolio model integrating additional information about the purchasing context to validate their feasibility and suitability. The fuzzy DSS is applied to a demonstrative case study of an American multinational company operating in the field of Electric Power Systems and Alternative Energy Systems.
\end{abstract}

Keywords: Decision support, Strategic Purchasing, Portfolio model, fuzzy logic, case study.

\section{Introduction}

The complexity of business decisions as concerning the definition of adequate purchasing strategies and the assessment of opportune Buyer-Supplier (B-S) relationships, does not allow for simple recommendations. In contrast with growing acceptance and usage of Purchasing Portfolio models (Kraljic 1983; Olsen and Ellram, 1997), a major and most severe criticism recently moved to their main rationale is the extreme simplification of the decision logic which is often based on just two basic dimensions (Gelderman, 2003; 2005; 2006).

By simplifying the issue of buyer-supplier relationships and other context-related dimensions, portfolio models fail to capture essential aspects, such as the impact of overall company business strategy, corporate purchasing policies, the context of networks (Dubois and Pedersen 2002), the interdependencies between products (Ritter 2000), and other relevant item features (e.g. product lifecycle) or the concern for a sustainable competitive advantage.

At the same time, purchasing portfolio models potentially suffer of arbitrariness and compensative processes due to related problems in item positioning. They are sensitive to the choice of the evaluation dimensions, factors and weights (Ramsay, 1996). Moreover, a large number of these variables are qualitative and need to be assessed subjectively by experts basing on their own experience or specific analysis of the business sectors. 
This paper proposes a fuzzy-based decision model to define appropriate and feasible strategic purchasing directions and action plans. Combining these kind of analysis with additional information, as for example about the supply market (the relative contractual power), supplier capabilities, company purchasing policies, the paper suggests an integrated decision logic enhancing practitioners in refining recommendations which are drawn on the traditional portfolio models by Kraljic (1983) and Olsen-Ellram (1997). Moreover, Fuzzy Set Theory is used in the Decision Support System in order to deal with the aggregation of different variables and often subjective information (e.g. linguistic variables vs numerical indicators, objective performance indexes vs subjective judgment) and to reproduce approximate, knowledge based and not-compensative reasoning.

\section{Research Objective}

Purchasing portfolio approach is usually a three step process finalized to manage supplier relationships. The first step generally address a product-based classification of components (Kralijc 1983, Olsen and Ellram, 1997; Kamann and Van Nieulande 2010), the second regards the analysis of suppliers and buyer supplier relationships (Olsen Ellram, 1997; Bensau, 1999; Caniel and Gelderman, 2007), the last step is decisional and draws on the propaedeutic information gathered to establish strategies and recommendations.

In this paper, we refer to the most known O-E model (Olsen and Ellram, 1997) which goes through the following 3 steps (Nellore and Soderquist, 2000):

1. analysis of the products and their classification according to the difficulty of managing the purchase situation and the importance of the purchase similarly to Kraljic (1983);

2. analysis of the supplier relationships required to deliver the products according to the Relative Supplier Attractiveness and B-S Strength of the Relationship (Olsen and Ellram, 1997); and

3. development of action plans in order to match the product requirements with the supplier relationships.

To successfully accomplish this process, decision makers have to arrange the decision logic integrating all the required information at the right step. This includes an appropriate selection of the critical decision dimensions and variables of analysis; a correct operationalization of the selected variables, related measurement and data collection; an effective item positioning (classification of goods-services et/or B-S relationships); and finally the integration of additional or complementary information for action validity check and synthesis of recommendations.

In this perspective, portfolio models, have been mostly criticized by different authors and perspectives both for their measurement concerns, and for their limited applicability in practice (Kamann 2000; Nellore and Soderquist, 2000; Dubois and Pedersen 2002). 
The present work focuses on the last three previous activities: it integrates in an overall process $\mathrm{K}$ and $\mathrm{O}-\mathrm{E}$ matrixes, it develops a fuzzy-based implementation of the O-E matrix (Olsen and Ellram, 1997) in order to deal with the problem of positing suppliers correctly starting from subjective judgments and weights; and finally, it validates and refines out coming recommendations suggesting additional information to be included in the decision process.

\section{Methodology}

\subsection{Model Architecture}

The proposed decision model consists of two main stages (Fig.1).

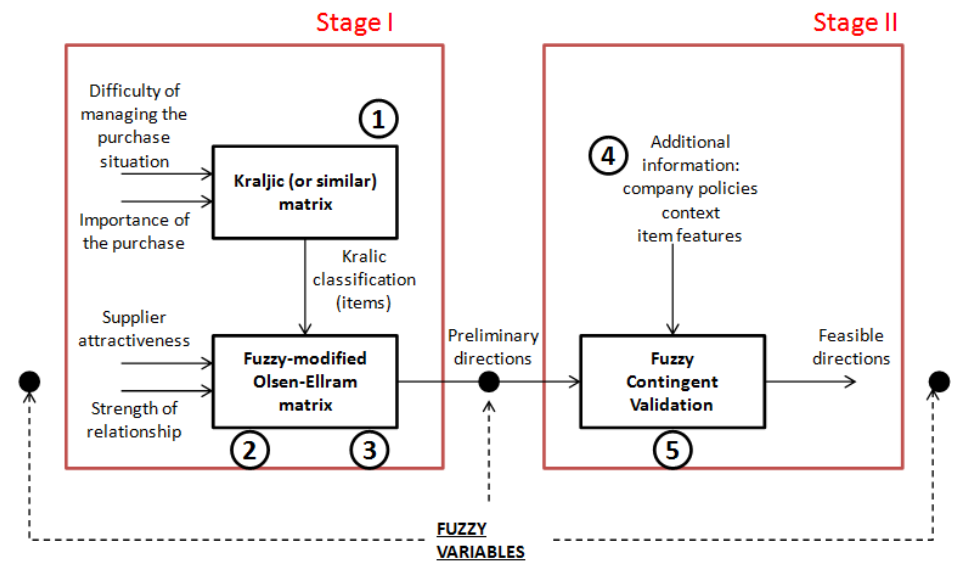

Fig. 1. A two stages (and five steps) decision logic

1. Firstly, it combines evidences of the current Buyer-Supplier relationships (according to a fuzzy implementation of the O-E matrix) with outputs from the analysis of purchasing items according to the Kraljic matrix (1983) perspective in order to define preliminary outputs (see step 1, 2, and 3 of O-E model).

2. In a second stage, instead, it completes the gathered information with additional considerations about specific company policies (e.g. localization: local vs global), purchasing item features (e.g. life cycle), and with other context related information about the purchasing situation (e.g. the relative contractual power between Buyer and Supplier, cross-supply level, level of shared investments on the suppliers/buyer side). This information is integrated into the decision model in order to provide a more effective and systematic validation of the outputs, and in case to refine recommendations basing on specific company contingencies to finally provide users with feasible and suitable action plans. In the steps 4 and 5, first the additional variables of analysis are selected and measured, and then preliminary outputs are refined accordingly by a fuzzy contingent validation. 
In order to face with the related complexity and uncertainty which is distinctive in dealing with information often subjective in nature and up to the knowledge and experience of respondents, we considered fuzzy logic as potential useful and flexible way to deal with the measurement of variables and the item positioning, as well as to arrange a not-compensatory decision logic and support user during the process. Thus, we adopted fuzzy variables in order to set up the input data to the O-E matrix (Olsen and Ellram, 1997) and in order to define the related outputs. At this stage of the decision process, information about the purchasing situation (provided by Kraljic portfolio analysis) are added and taken into consideration within the Fuzzy Inferential System (FIS), in order to define preliminary directions. Fuzzy set theory is also adopted at the stage 2 to characterize the preliminary and final model outputs, the additional information here included and the overall decision logic.

\subsection{Inputs, Outputs and Additional Control Variables}

Inputs: Variables are defined according to Olsen and Ellram (1997) model. Two macro dimensions are adopted for each matrix as specified previously. A number of factors and sub-factors was assessed coherently in order to provide a complete measurement scale.

Outputs: The model presents a more analytic (multi-input-multi-output model) output structure in respect to the most traditional approaches. This is in order to offer practitioners better precisions and an easier interpretation of indications. Output variables (Table 1) are drawn, refined and completed accordingly the main directions provided by Olsen and Ellram (1997) and subsequent criticism (Kamann 2000; Nellore and Soderquist, 2000; Dubois and Pedersen 2002). Variables offer information about both strategic directions and macro actions to be implemented.

Table 1. O-E Matrix Outputs

(adapted from: Olsen and Ellram, 1997)

\begin{tabular}{l}
\hline O-E directions \\
\hline 1. Strengthen the relationship \\
(or diversify) \\
2. Improve supplier attractiveness \\
and/or relation performance \\
3. Resource (allocation) level \\
4. Investment level \\
5. Timing horizon (short/long term) \\
\hline
\end{tabular}

Table 2. Additional Information

\begin{tabular}{l}
\hline $\begin{array}{l}\text { Additional/context } \\
\text { information }\end{array}$ \\
\hline 1. Budget constrains \\
2.Uniformity constrains (Global vs \\
local purchasing; single vs multi \\
sourcing; Sustainability/Ethic; other \\
purchasing policies) \\
3. Life cycle stage \\
4. Concurrent/shared investments \\
5. Allocated resource \\
6. Cross-supply level
\end{tabular}

Additional information (or control variables): Feasible and suitable actions should be evaluated in the light of some critical context-related information as for example that related to existing company constrains (budget, policies and other limitations to the supply base) or other influencing factors. This is particularly true when purchasing portfolio models are used to draw strategic directions at the Business Unit level since they usually need to be aligned with the Corporate directives. 
In order to accomplish this last purpose, in the second decision step, the model integrates a number of variables (mostly subjective data) provided directly by the decision maker in order to validate the feasibility and suitability of preliminary directions. Specifically, two classes of information are considered: the first is related to "Corporate constrains", mainly budget and uniformity constrains (i.e. standardization and homogeneity in the supply process). These factors may limit investments (capital budget decisions), as well as influence criteria for the supplier selection process (the type of B-S relation, information exchange, integration level), and also impact on purchasing policies (sustainability, ethic, local/global and multi/single sourcing policies). The second class of information, instead, includes context-related factors which might limit the appropriateness and feasibility of proposed recommendations: the product life cycle stage, the level of current/shared investments (i.e. technology, specific asset, switching costs), the company resources currently allocated to the B-S relation, the company's network position, the level of cross supply. Details of selected variables are reported in table 2 .

As an example, the product life cycle (plc) may affect and influence the out-coming strategic direction and action plans since the objectives of the B-S relations, the desirable performance, and also the available resource and risk change accordingly to the different plc-stages. Thus, an effective decision model should take into account this factor in order to provide really suitable recommendations.

\section{Case Study}

\subsection{Context}

The method was developed and applied to the case of a multinational company in the Power-electronic market. In the case study we refer to a specific implementation of the presented decision model in order to demonstrate the applicability of the proposed approach. A team of experts from the Company and University was built to run the project. For the purpose of this work, we assumed that the analysis of the purchasing situation (through the Kraljic matrix) was previously and adequately performed coherently with the evaluation objectives, as also we hypothesize that the adopted measures and the data collection process have been appropriately carried out. As a consequence, the focus of the paper is limited to the investigation of the Buyer-Supplier relationships (by O-E matrix) and on the additional information to be included in the decision logic (rules) to draw feasible and suitable strategies and action plans.

\subsection{Data Collection}

A highly qualified panel of academic experts and practitioners from the Sourcing and Purchasing functions was formed to select the most appropriate (and at the same time available) measures according to the $\mathrm{OE}$ dimensions, to define the relative weights, and to finally characterize the purchasing items/suppliers accordingly. Two questionnaires were assessed to obtain data. The first questionnaire addressed the importance 
scores of the selected attributes according to an AHP procedure while the second one was used for finding out the performance scores of suppliers. Respondents were asked to indicate the degree to which they agree with each statement on a 5-point fuzzy linguistic scale. A Delphi-based process was also adopted in order to achieve convergence of the experts' judgments.

Company experts was also involved in the DSS assessment and setting as concerning the definition of FIS rules, and the final model validation mostly by semistructured interviews, focus group and observation on the field.

\subsection{Model Design}

The FIS design usually goes through the following steps:

\section{Membership function design}

In order to construct the fuzzy model, a number of semi-structured interviews was held. An open-ended questionnaire was used to conduct the interviews, including a set of questions to discuss the fuzzy variables and define their labels (linguistic values) and determine the fuzzy ranges of each value label whereas data were not available.

The model consists of input variables which attain the two main OE dimensions: Supplier Attractiveness and Strength of the Relationship. Some building factors are selected in collaboration with the company experts in order to assure data/knowledge availability to users and relevance of information into the company decision process. All the input variables are provided as 5-point linguistic scale by triangular fuzzy numbers. Then, factors are aggregated following a Ordered Weighted Average (OWA) procedure (Yager, 1988) considering weights which were defined by the Sourcing, Logistic and Quality managers according to a Delphi-AHP procedure (Okoli and Pawlowski, 2004; Saaty, 2006). An order relevant input to the model is the Item classification accordingly to Kraljic matrix, which is here modeled as a rule discriminator value (i.e. it modifies the rule base).

Preliminary outputs of the model as in Table 1 are modeled as a three level fuzzy scale. Specifically, they indicate to how extent the management should plan actions for strengthening the relation (high) or diversifying the supplier base (low); improving supplier attractiveness and/or performance; to how extend investing new assets/technology; allocating new resources into the relation; and also which is the time horizon of the relationship.

Control variables included in the second step of the decisional model are interpreted, as previously stated, as context-related variables or company constrains which may be due to the corporate policies and/or available budget (Table 4). They are modeled as 5-point linguistic scale by triangular fuzzy numbers as well.

\section{Rule settings}

In setting the decision rules of the FIS we used two common approaches in literature:

- Adopting a normalized or standard rule-base: firstly, we are here drawing on directions by the $\mathrm{K}$ and $\mathrm{OE}$ portfolio models. 
- Codifying the experience and intuition of experts: rules were derived from the managerial procedures, from interviews using a carefully designed questionnaire, or often observing an actions to deduce if-then type rules. In this case, the approach is used to further investigate suitability and validity of preliminary OE direction according to the additional information provided.

The FIS uses the Mamdani inference method, once all the screening heuristics have been identified, they have to be transformed into proper fuzzy IF-THEN rules. More than 100 rules were finally defined and implemented in a FIS by the support of the Matlab Fuzzy rules editor toolbox.

For a purpose of clarity and due to space limitation, here we just present how the model works for a specific combination purchasing item/supplier. Once input factors are defined and evaluated, the fuzzy OE matrix is assessed. Judgments were aggregated into a single fuzzy index by a ordered weighted average (OWA). As a result, this procedure allows to assign a supplier to more than a single quadrant in the matrix according to a different membership degree and a fuzzy borderline which make classification more flexible and less restrictive (Fig. 2).

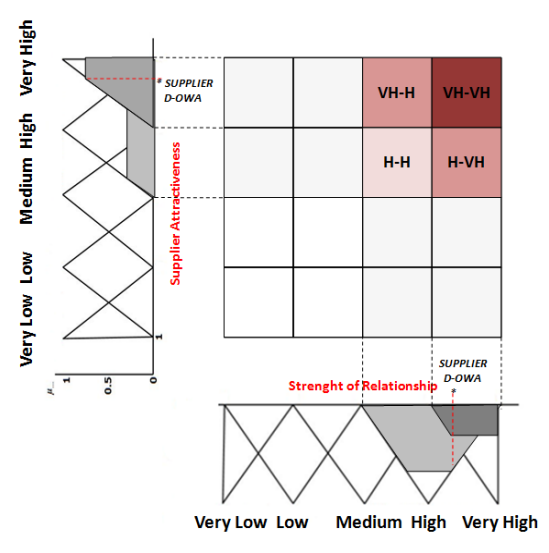

Fig. 2. Positioning on the fuzzy O-E matrix

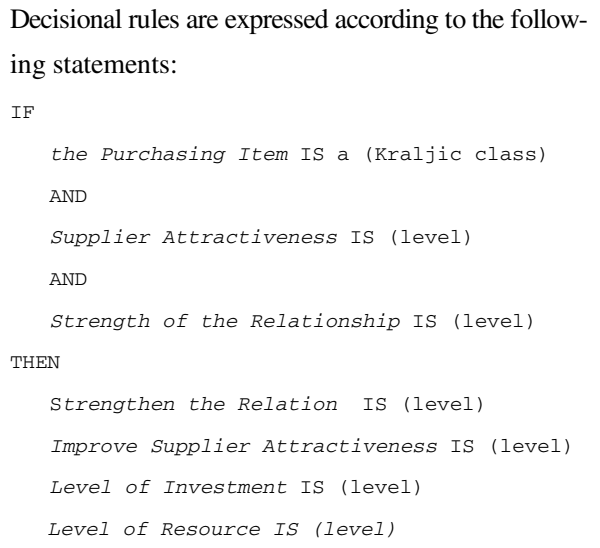

Preliminary outcomes are finally refined in a second step considering the company constrains. The decision logic and fuzzy rules were set similarly to the previous ones.

\section{Validation and/or Sensitivity Analysis}

Model validation is currently in progress so that the DSS is still under refinement and liable to modifications. Managers and senior buyers from the company implementation team were involved in the process in order to test the proper operation of the fuzzy systems. With the support of experts, the validation of a number of cases is programmed in order to check model Completeness, Consistency, Redundancy and Interaction. 


\section{Conclusion}

The main result of this work was the development of a decision support system enhancing users to effectively integrate traditional purchasing portfolio approaches with additional strategic oriented priorities/information in defining purchasing directions and action plans. A fuzzy-based decision model is proposed and implemented. The decision process gets inputs from the Kraljic matrix and draws directives on the traditional OE portfolio model integrating additional information about the purchasing context (company strategy, supply market, etc) to validate feasibility and suitability. The fuzzy DSS is applied to a demonstrative case study of an American multinational company operating in the field of Electric Power Systems and Alternative Energy Systems.

The DSS particularly helps decision makers in facing a number of common issues affecting the use of portfolio approaches, e.g. the Olsen-Ellram matrix, as such as:

- To deal with the assessment of subjective variables in order to get a correct and not arbitrary positioning of suppliers;

- To define appropriate rules for drawing feasible and suitable strategic directions and actions plans keeping into account additional information about strategic priorities or company policies.

\section{References}

1. Gelderman, C.J., Van Weele, A.J.: Handling MeasurementIssues and Strategic Directions in Kraljic's Purchasing Portfolio Model. Journal of Purchasing and Supply Management 9(56), 207-216 (2003)

2. Gelderman, C.J., Van Weele, A.J.: Purchasing Portfolio Models: A Critique and Update. The Journal of Supply Chain Management (Summer 2005)

3. Gelderman, C.J., Semeijn, J.: Managing the global supply base through purchasing portfolio management. Journal of Purchasing \& Supply Management 12(4), 209-217 (2006)

4. Kraljic, P.: Purchasing Must Become Supply Management. Harvard Business Review 61(5), 109-117 (1983)

5. Okoli, C., Pawlowski, S.D.: The Delphi method as a research tool: An example, design considerations and applications. Inform. Manage. 42, 15-29 (2004)

6. Olsen, R.F., Ellram, L.M.: A Portfolio Approach to Supplier Relationships. Industrial Marketing Management 26(2), 101-113 (1997)

7. Yager, R.R.: On Ordered Weighted Averaging Aggregation Operators in Multi-criteria Decisions. IEEE Trans. on Systems, Man, and Cybernetics. 8(1), 183-190 (1988)

8. Zimmermann, H.J.: Fuzzy Sets, Decision Making, and Expert Systems, International Series in Management Science and Operations Research, Ignizio, University of Huston USA (1993) 